\title{
一次爆炸焊接制备铝/镁/铝合金复合板的数值模拟"
}

\author{
魏 屹 $1,2,3$ 王永祯 ${ }^{2}$ 王文先 ${ }^{1,2,3}$ 张婷婷 $1,2,3$ 间志峰 $1,2,3$
}

(1. 先进镁基材料山西省重点实验室 太原 030024;

2. 太原理工大学材料科学与工程学院 太原 030024 ;

3. 新材料界面科学与工程省部共建教育部重点实验室 太原 030024)

摘要: 爆炸焊接过程具有瞬时、极速和复杂物理化学反应的特点, 数值模拟是一种重要且有效指导和优化爆炸焊接试验的方 法。提出一次爆炸焊接方法制备铝/镁/铝合金复合板, 采用 SEM 和 EDS 表征复合板结合界面形貌及组织成分, 研究发现铝/ 镁/铝合金复合板界面形成明显的波形界面，且结合界面处发生明显的扩散行为。通过数值模拟的方法分析爆炸焊接过程中复 合板界面的应力应变场分布和温度场分布, 结果表明复合板结合界面的连接机理是压力焊、熔化焊和扩散焊综合作用的结果。 所得结果有利于指导一次爆炸焊接成型制备多层复合板及连接界面结合机理的研究。

关键词: 爆炸焊接; 铝/镁/铝复合板; 数值模拟; 连接机理

中图法分类号: TG156

\section{Numerical Simulation of Al/Mg/Al Composite Plates Fabricated by Explosive Welding in One Stage}

\author{
WEI Yi $^{1,2,3}$ WANG Yongzhen $^{2}$ WANG Wenxian ${ }^{1,2,3}$ ZHANG Tingting ${ }^{1,2,3}$ YAN Zhifeng ${ }^{1,2,3}$ \\ (1. Shanxi Key Laboratory of Advanced Magnesium-based Materials, Taiyuan 030024;
}

2. College of Materials Science and Engineering, Taiyuan University of Technology, Taiyuan 030024;

3. Key Laboratory of Interface Science and Engineering in Advanced Materials,

Ministry of Education, Taiyuan 030024)

\begin{abstract}
Explosive welding has characteristics of instantaneous, rapid and complicated reactions. The numerical simulation is important and effective method to research explosive welding. The method of explosive welding in one stage is used to fabricate the $\mathrm{Al} / \mathrm{Mg} / \mathrm{Al}$ composite plates. The SEM and EDS are adopted to research the morphology and the component of interface. The bonding interface presents a periodical wave and the diffusion phenomena is detected at interface. The bonding interface has serious plastic deformation because the serious pressure on the interface in the processes of explosive welding. The numerical simulation tests are used to research the distribution of temperature, stress and strain in the processes of explosive welding. The temperature at the interface is much more than the melting points of AZ31B and 6061. The results show that the bonding mechanism is interaction of pressure welding, diffusion welding and local melting welding. The research of explosive welding in one stage and the bonding mechanism of interface can be guided.
\end{abstract}

Key words: explosive welding; $\mathrm{Al} / \mathrm{Mg} / \mathrm{Al}$ composite plates; numerical simulation tests; bonding mechanism

\section{0 前言}

镁合金材料由于具有轻质高强和资源丰富的优 势，已成为继钢铁、铝合金材料之后的第三大金属 工程材料。但由于其韧性低、抗腐蚀能力差等缺点,

* 国家自然科学基金(51375328、51805359)和中国博士后科学基金面上项 目(2018M631772)资助项目。20180921 收到初稿, 20190328 收到修改稿
严重制约了其优势的发挥和应用。而铝合金具有较 高的㓞性以及抗腐蚀能力，且铝合金是仅次于镁合 金的低密度轻金属结构材料 ${ }^{[1-3]}$ 。因此，提出在镁合 金表面覆铝合金的方法来制备层状复合材料, 以改 善镁合金材料的耐腐蚀性能 ${ }^{[4-6]}$ 。

爆炸焊接方法是一种重要的层状复合板制备方 法, 特别适用于一般熔焊方法难以焊接的异种材料 的连接 ${ }^{[7]}$ 。目前, 镁基合金的爆炸焊接研究主要集 中在两层复合板的研究 ${ }^{[8-10]}$ 。课题组张楠等 ${ }^{[11]}$ 利用 
爆炸焊接的方法成功的制备了的复合板材, 武佳琪 等 ${ }^{[12]}$ 利用爆炸焊接的方法制备了镁合金 AZ31B 与 钛合金 TA2 的复合板, 张婷婷等 ${ }^{[13]}$ 研究了镁合金 AZ31B 与铝合金 AA6061 爆炸焊接复合板界面的结 合机理。

相比于双层结构的异种层状复合板, 对称结构 的三明治复合板在二次加工时, 具有更好的协调变 形能力 ${ }^{[14]}$, 且对基板 (中间层) 有更好的防护作用。 因此, 采用爆炸焊接法制备三明治结构的层状复合 板显得尤为重要。目前, 采用爆炸焊接方法制备三 明治结构的层状复合材料常常采用多次爆炸焊接复 合成型的工艺, 采用该工艺制备复合材料时, 由于 受第一次爆炸焊接成型的影响, 复合板连接界面不 可避免地会出现一些未结合等缺陷。基于此, 本文 提出一次爆炸焊接法制备铝/镁/铝合金三明治结构 的层状复合板, 采用 SEM 和 EDS 对界面结合形貌、 组织成分进行了分析研究; 采用数值模拟对复合板 结合界面处压力分布、应力应变场分布和温度场分 布进行了分析。

本文所采用的数值模拟方法是利用 AUTODYN 模块来模拟 $\mathrm{Al}-\mathrm{Mg}-\mathrm{Al}$ 三层复合板爆炸焊接一次成 型的过程, 模拟过程中采用的是光滑粒子流体动力 学方法 (SPH)。在模型中选用 Mie-Gruneisen 状态方 程作为铝/镁合金在数值模拟过程中的本构方程, 该 状态方程适用于表征材料在大变形状态下的动力学 行为。选用的 Steinberg-Guinan 强度模型能有效地 描述材料在大变形情况下的塑性变形行为 ${ }^{[15-17]}$ 。采 用爆轰波的 C-J 理论来表示炸药的爆轰作用, 而爆 轰产物的状态方程采用 JWL(Jones-Wilkins-Lee)状 态方程。

\section{1 试验材料、方法及模型建立}

\section{1 数值模拟}

铝/镁/铝合金爆炸焊接数值模拟初始示意图如 图 1 所示。所选材料及炸药参数如表 $1 、 2$ 所示。镁

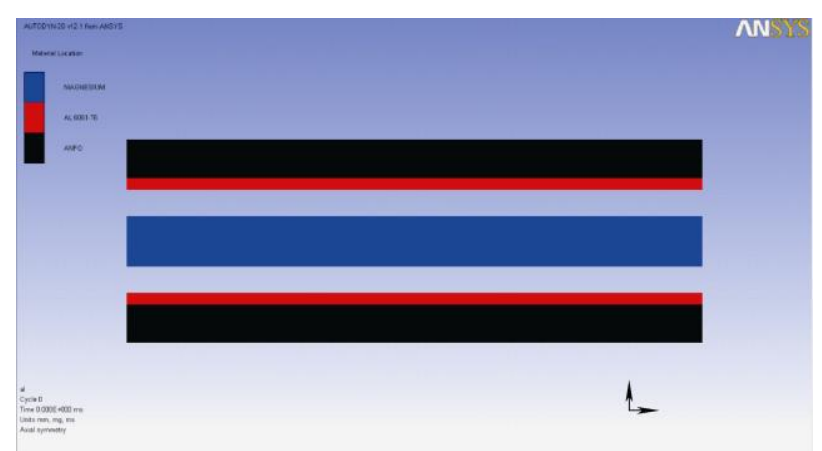

图 1 爆炸焊接数值模拟建模图
合金 AZ31B 基板尺寸为 $40 \mathrm{~mm} \times 10 \mathrm{~mm}$, 铝合金 $A A 6061$ 覆板尺寸为 $40 \mathrm{~mm} \times 2 \mathrm{~mm}$, 两侧炸药厚度 均为 $6 \mathrm{~mm}$ 。

表 1 数值模拟试验中选用的材料参数

\begin{tabular}{|c|c|c|c|c|c|}
\hline 材料 & $\begin{array}{c}\text { 密度 } \\
\rho_{\mathrm{m}} /\left(\mathrm{g} / \mathrm{cm}^{3}\right) \\
\end{array}$ & $\begin{array}{l}\text { 熔点 } \\
T_{\mathrm{m}} / \mathrm{K} \\
\end{array}$ & $\begin{array}{c}\text { 剪切模量 } \\
G / \mathrm{GPa} \\
\end{array}$ & $\begin{array}{c}\text { 屈服应力 } \\
Y / \mathrm{MPa}\end{array}$ & $\begin{array}{c}\text { 最大屈服应力 } \\
Y_{\mathrm{m}} / \mathrm{MPa} \\
\end{array}$ \\
\hline AZ31B & 1.78 & 923 & 16.5 & 220 & 480 \\
\hline 6061 & 2.703 & 934 & 26.9 & 290 & 680 \\
\hline \multicolumn{6}{|c|}{ 表 2 数值模拟过程中的炸药参数 } \\
\hline \multirow{2}{*}{ 材料 } & \multirow{2}{*}{$\begin{array}{c}\text { 密度 } \\
\rho_{\mathrm{m}} /\left(\mathrm{g} / \mathrm{cm}^{3}\right)\end{array}$} & \multirow{2}{*}{\multicolumn{2}{|c|}{$\begin{array}{c}\text { 炸药爆速 } \\
D /(\mathrm{m} / \mathrm{s})\end{array}$}} & 爆轰压力 & 炸药能量 \\
\hline & & & & $P / \mathrm{GPa}$ & $E /\left(\mathrm{GJ} / \mathrm{m}^{3}\right)$ \\
\hline 铵油炸药 & 0.931 & & 2500 & 5.15 & 2.484 \\
\hline
\end{tabular}

\section{2 试验材料}

本文选用基板为 $600 \mathrm{~mm} \times 300 \mathrm{~mm} \times 10 \mathrm{~mm}$ AZ31B 镁合金, 覆板为 $600 \mathrm{~mm} \times 300 \mathrm{~mm} \times 2 \mathrm{~mm}$ 的 6061 铝合金, 其化学成分的质量分数如表 3 所示。

表36061 铝合金和 AZ31B 镁合金化学成分的质量分数(\%)

\begin{tabular}{cccccccc}
\hline 材料 & $\mathrm{Mn}$ & $\mathrm{Mg}$ & $\mathrm{Zn}$ & $\mathrm{Ti}$ & $\mathrm{Si}$ & $\mathrm{Fe}$ & $\mathrm{Al}$ \\
\hline AZ31B & 0.63 & Bal. & 1.10 & - & 0.10 & 0.005 & 3.02 \\
6061 & 0.15 & $0.8 \sim 1.2$ & 0.25 & 0.15 & $0.4 \sim 0.8$ & 0.7 & Bal. \\
\hline
\end{tabular}

\section{3 试验方法}

爆炸焊接实际试验过程采用平行放置法, 选用 爆速为 $2500 \mathrm{~m} / \mathrm{s}$ 的铵油炸药(ANFO) $10 \mathrm{~mm}$ 进行爆 炸焊接, 基覆板间隙为 $4 \mathrm{~mm}$, 爆炸焊接前先用砂纸 将基覆板表面打磨干净。爆炸焊接方法示意图如图 2 所示。
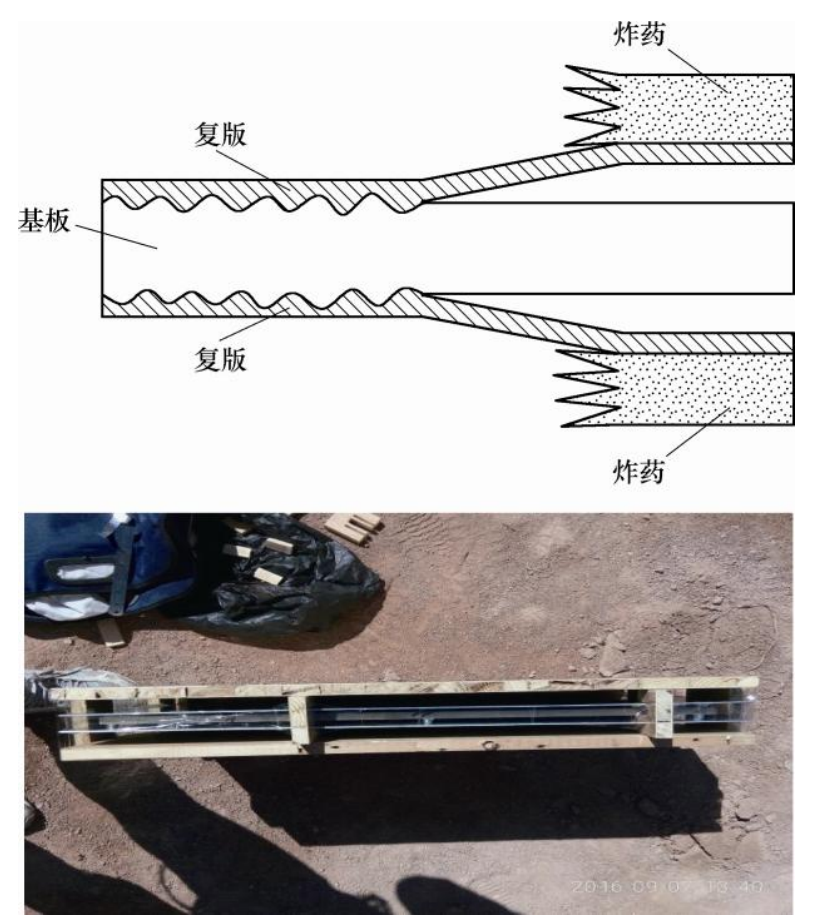

图 2 铝/镁/铝合金复合板一次爆炸焊接方法示意图 


\section{2 试验结果与讨论}

\section{1 数值模拟结果及分析}

图 3 所示为铝/镁/铝合金三层复合板爆炸焊接 数值模拟过程, 得到的复合板波形界面形貌以及产 生的射流如图 4 所示。

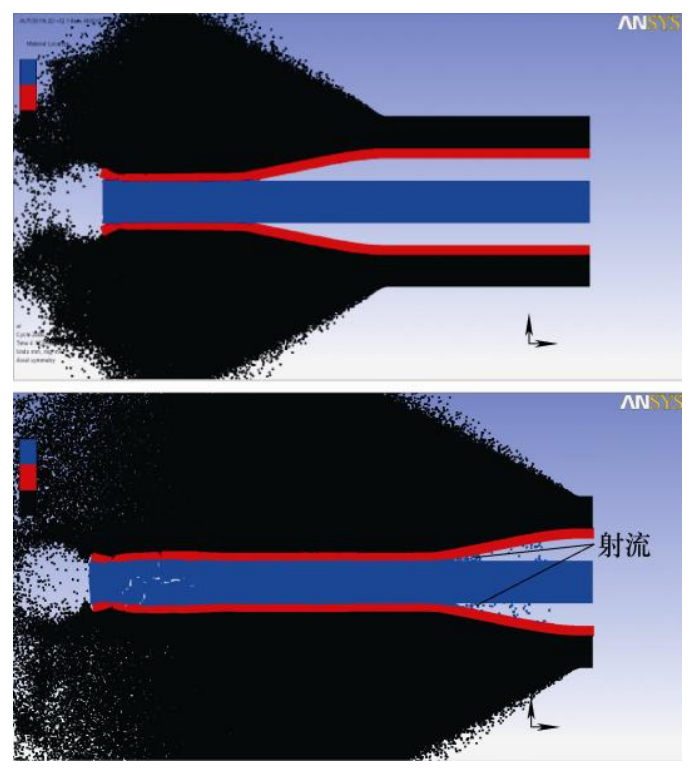

图 3 爆炸焊接数值模拟过程图

由图 3、4 所示的数值模拟过程和结合界面形 貌可知：爆炸焊接过程中产生了明显的射流且形 成了波形界面。在爆炸焊接过程中, 射流的形成 对于焊接成功与否以及焊接质量起到了重要的影 响。射流的产生不但可以对复合板表面进行清理 作用, 而且波形形貌的产生也与射流在爆炸焊接 过程中被捕获有关。因此, 射流的产生是爆炸焊 接成功的一个重要标志 ${ }^{[18-23]}$ 。一般而言, 爆炸焊 接过程中要产生射流现象, 碰撞点处的压力需要 达到材料静强度的十倍以上, 这也意味着覆板材 料需要达到一个极大的碰撞速度。理论上, 不论 碰撞角度如何, 射流产生的速度至少不能小于碰 撞点移动速度, 否则便无法脱离碰撞点区域从而 产生射流。复板平行放置时, 碰撞点移动速度即 为炸药爆速 ${ }^{[21]}$, 爆炸焊接过程中界面处的粒子速 度分布图如图 5 所示, 可以看出射流粒子速度均 大于炸药爆速。由数值模拟结果可知: 射流绝大 部分由镁合金提供, 铝合金量提供较少, 这是由 于在爆炸焊接过程中, 密度更小, 强度更低的材 料更容易产生射流 ${ }^{[16]}$ 。从波形形貌及射流现象可 以看出, 基板与覆板之间达成了良好的结合。

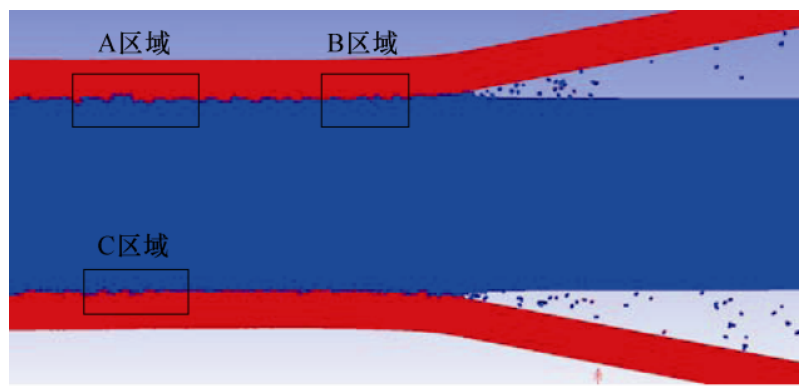

(a) 宏观图

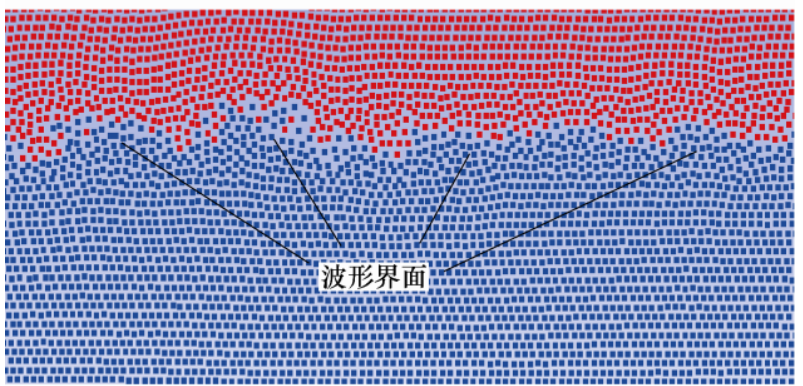

(b) A区域局部放大图

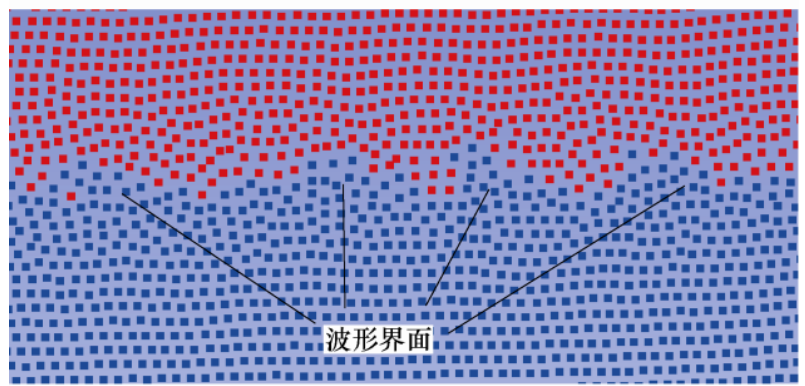

(c) B区域局部放大图

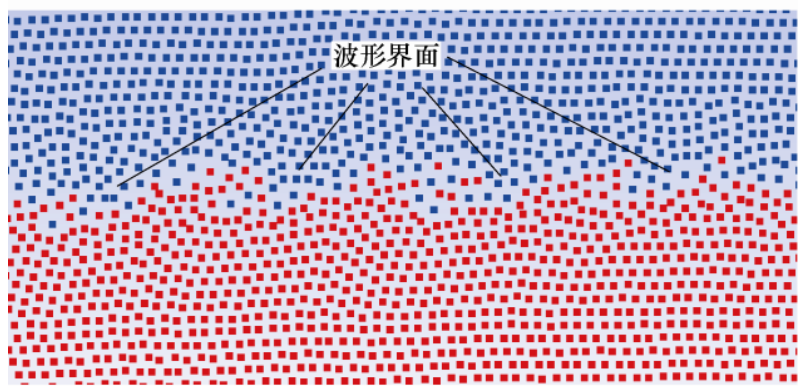

(d) C区域局部放大图

图 4 爆炸焊接数值模拟界面示意图

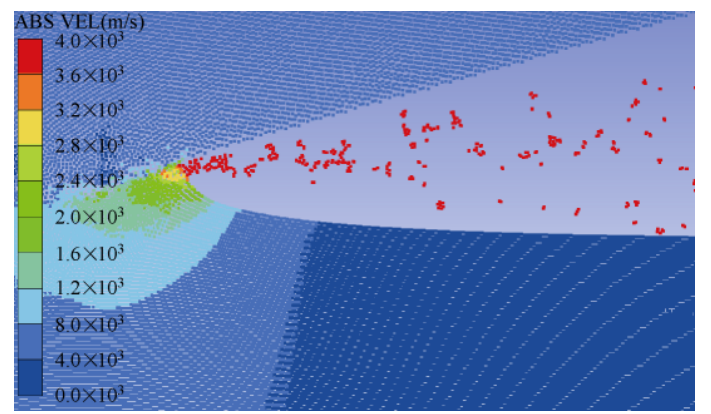

图 5 界面处的粒子速度分布图

图 6 所示为数值模拟过程中复合板界面的应力 曲线，分别在基板与覆板碰撞表面取点。镁合金和 铝合金的屈服强度分别是 $0.19 \mathrm{GPa}$ 和 $0.12 \mathrm{GPa}$ 。从 
应力曲线及应力分布情况中可以看出, 复合板界面 处受到极大的应力作用, 铝合金一侧所受压力可以 达到 $34 \mathrm{GPa}$ 以上, 镁合金一侧所受压力也可以达到 $29 \mathrm{GPa}$ 左右, 远远大于材料的屈服强度。因此, 结 合界面处的压力远大于材料的屈服强度是导致铝/ 镁结合界面处形成波形界面的主要原因 ${ }^{[17]}$ 。

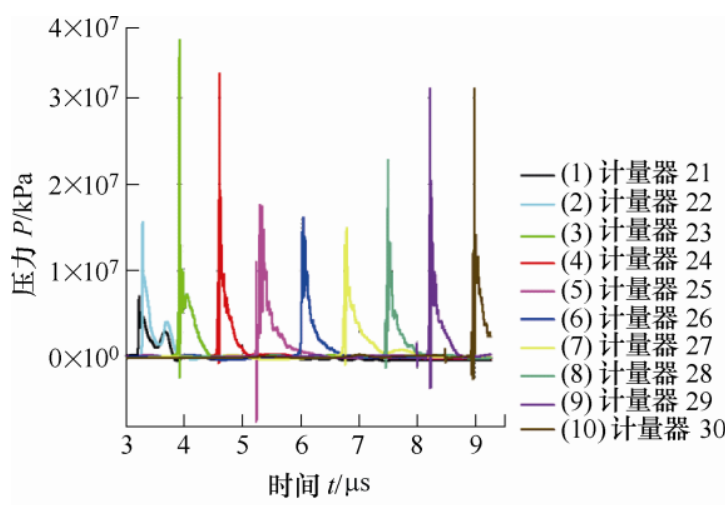

(a) 铝合金一侧

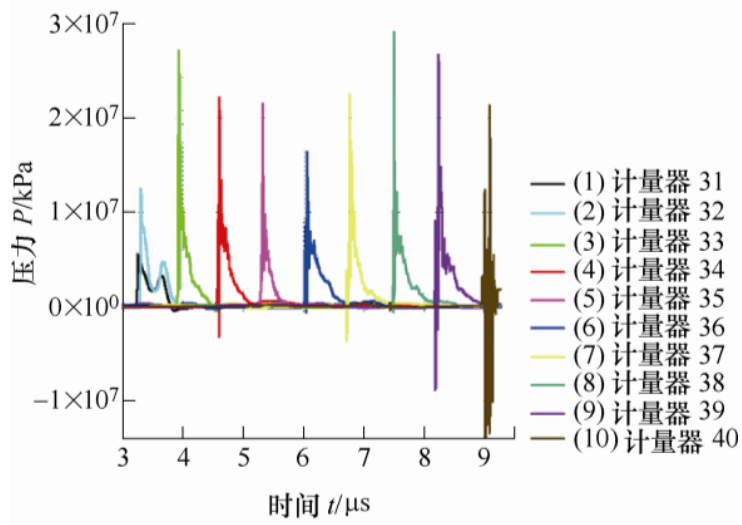

(b) 镁合金一侧

图 6 爆炸焊接过程复合板界面应力曲线

图 7、8 为数值模拟过程中复合板界面的应变曲 线及分布情况。从图 7、8 可知, 在碰撞压力的作用 下, 基板与覆板的塑性变形行为非常明显, 并在界 面接合区域产生一条狭窄的塑性变形带。从板材表 面所取点的应变曲线中可以看到镁合金基板的最大 应变达到了 $140 \%$, 而铝合金覆板的最大应变也达 到了 $120 \%$ 。

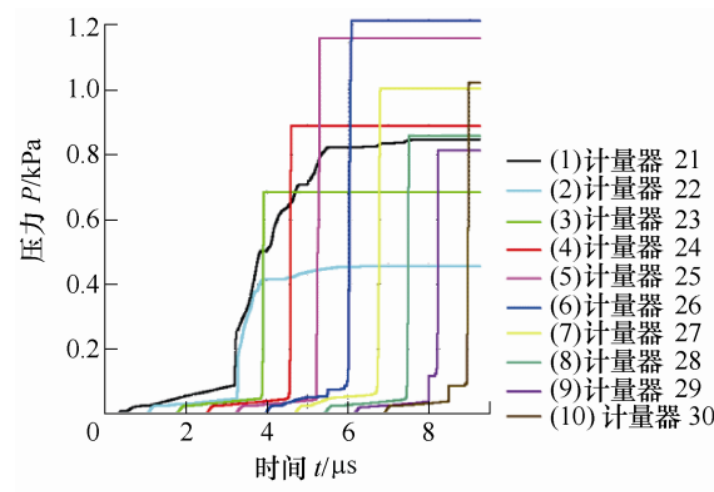

(a) 铝合金一侧

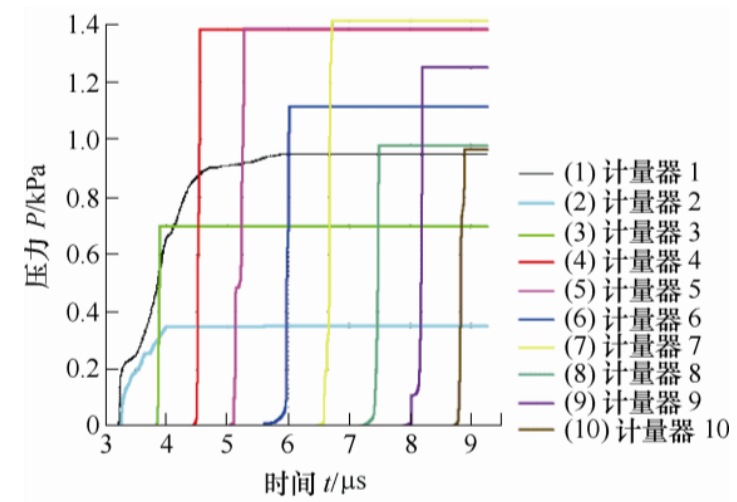

(b) 镁合金一侧

图 7 爆炸焊接过程复合板界面应变曲线

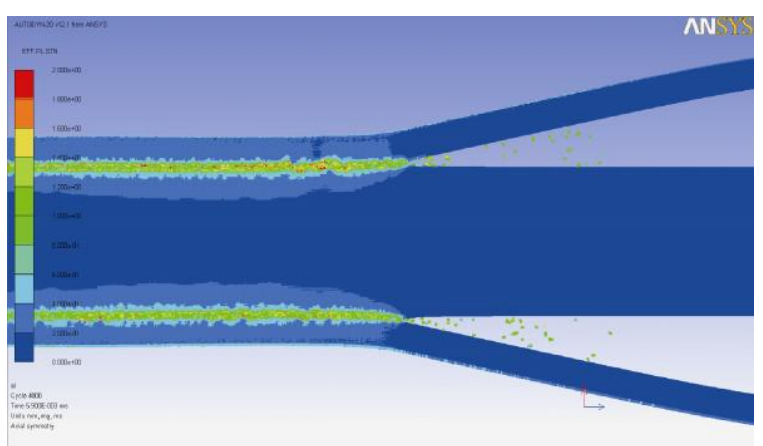

图 8 复合板界面处应变分布图

图 9 为数值模拟过程中温度分布图。可以看到 在爆炸焊接过程中界面处温度可以超过 $1200 \mathrm{~K}$, 远 高于镁合金 AZ31B 的熔点 $923 \mathrm{~K}$ 以及铝合金 6061 的熔点 $933 \mathrm{~K}$ 。由此可知, 铝/镁/铝合金爆炸焊接 过程中界面处存在局部熔化现象。

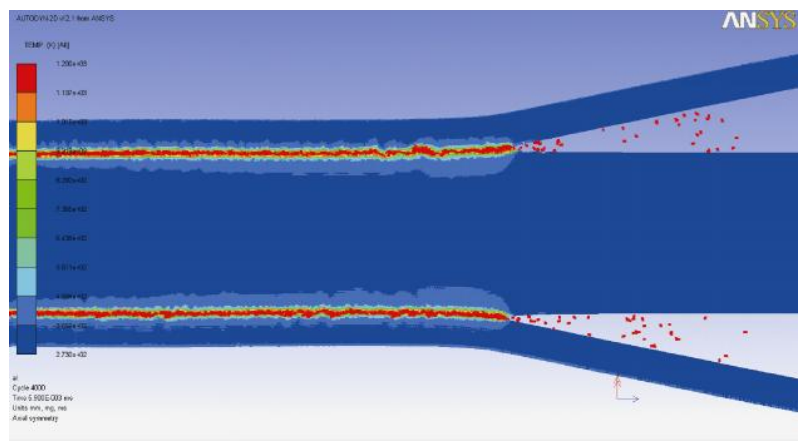

图 9 数值模拟过程温度分布图

\section{2 铝/镁/铝合金爆炸焊接复合板接合界面形貌}

铝/镁/铝复合板结合界面形貌如图 10 所示。 由图可知, 复合板的两侧铝/镁结合界面均呈现明 显的波形界面形貌，且结合界面处未出现未结合、 局部熔化等缺陷。对该结合界面处进一步采用 EDS 线扫描分析(图 11), 结果表明铝/镁结合界 面处发生了明显的扩散现象，扩散层的厚度大约 为 $1.5 \mu \mathrm{m}$ 。而线扫描曲线并没有存在明显的阶梯 现象，因此扩散并没有生成金属间化合物这种硬 脆相 ${ }^{[13]}$ 。 


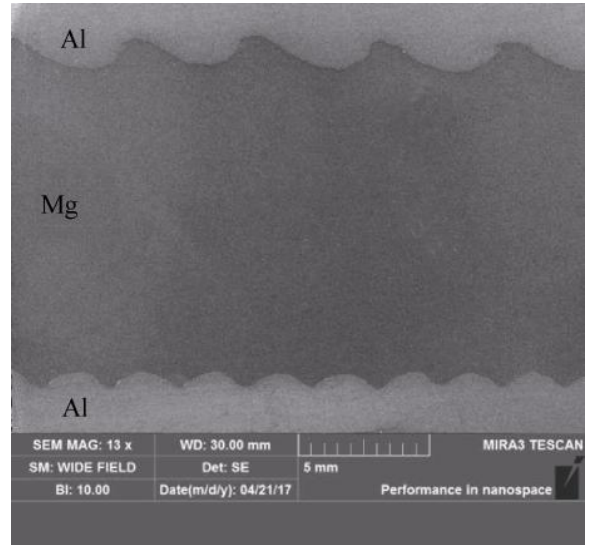

图 10 铝/镁/铝合金爆炸焊接复合板接合界面波形形貌

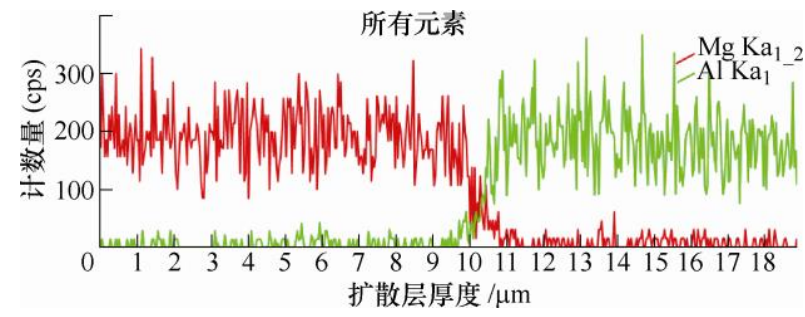

图 11 镁铝界面处线扫描结果

\section{3 界面结合机理分析}

综合分析铝/镁/铝三层复合板 SEM、EDS 试验 结果和数值模拟结果可知: 铝/镁/铝合金爆炸焊接 界面结合是由压力焊机理、熔化焊机理和扩散焊综 合作用的结果。

(1) 压力焊机理: 根据数值模拟对结合界面处 的压力分布可知, 复合板界面处受到远大于铝合金 和镁合金材料的屈服强度，使得界面处材料发生明 显的塑性变形，形成了波形界面;

(2) 熔化焊机理：根据铝/镁结合界面处的温度 场分布可知, 界面处在爆炸焊接过程中连接界面形 成了一层高温熔化层, 且远高于两侧板材的熔点, 使得界面处材料发生局部熔化形成了冶金结合;

(3) 扩散焊机理: 从复合板界面处的线扫描结 果可以看出, 复合板界面存在一定的铝镁元素扩散 现象，且扩散现象有利于复合板界面的结合。

\section{3 结论}

(1) 数值模拟结果表明: 爆炸焊接过程中, 连 接界面形成了明显的射流; 结合界面处的压力分布 远大于材料的屈服强度是形成波形界面的主要原 因; 结合界面处形成了高温熔化层导致了连接界面 局部熔化层的形成。

(2) SEM 和 EDS 试验结果表明: 复合板结合界 面均形成了明显的波形界面, 且连接界面发生了一 定的扩散, 扩散层厚度大约为 $1.5 \mu \mathrm{m}$ 。
（3）综合试验表征和数值模拟结果发现: 铝/镁/ 铝复合板结合界面连接的主要原因可归结为压力 焊、熔化焊和扩散焊综合作用的结果。

\section{参 考 文 献}

[1] HIRSCH J, Al-SAMMAN T. Superior light metals by texture engineering: optimized aluminum and magnesium alloys for automotive applications[J]. Acta Materialia, 2013, 61(3): 818-843.

[2] LIU Fencheng, LIANG Wei, LI Xianrong, et al. Improvement of corrosion resistance of pure magnesium via vacuum pack treatment[J]. Journal of Alloys and Compounds, 2008, 461(1-2): 399-403.

[3] WEI Fangfang, ZHANG Wei, ZHANG Tao, et al. Effect of variations of $\mathrm{Al}$ content on microstructure and corrosion resistance of PEO coatings on $\mathrm{Mg}-\mathrm{Al}$ alloys $[\mathrm{J}]$. Journal of Alloys and Compounds, 2017, 690(5): 195-205.

[4] PARDO A, CASAJUS P, MOHEDANO M. Corrosion protection of $\mathrm{Mg} / \mathrm{Al}$ alloys by thermal sprayed aluminum coatings. Applied Surface Science 2009, 255: 6968-6977.

[5] LEO P, RENNA G, CASALINO G, et al. Effect of power distribution on the weld quality during hybrid laser welding of an Al-Mg alloy[J]. Optics \& Laser Technology, 2015, 73: 118-126.

[6] WANG Peng, HU Shengsun, SHEN Junqi, et al. Effects of electrode positive/negative ratio on microstructure and mechanical properties of $\mathrm{Mg} / \mathrm{Al}$ dissimilar variable polarity cold metal transfer welded joints[J]. Materials Science \& Engineering A, 2015, 652: 127-135.

[7] AKBARI-MOUSAVI S A A, Al-HASSANI S T S, ATKINS A G. Bond strength of explosively welded specimens[J]. Materials and Design, 2008, 29(7): 1334-1352.

[8] 张越举, 杨旭升, 李晓杰, 等. 钛/钢复合板爆炸焊接 实验 $[\mathrm{J}]$. 爆炸与冲击，2012，32(1)：103-107.

ZHANG Yueju, YANG Xusheng, LI Xiaojie, et al. An experimental research on explosion welding of titanium/ steel clad plate[J]. Explosion and Shock Waves, 2012, 32(1): 103-107

[9] 陆明，王耀华，尤俊，等. 工具钢/Q235 复合板爆炸焊 接试验及性能研究 [J]. 焊接学报, 2001, 22(4): 47-50. LU Ming, WANG Yaohua, YOU Jun, et al. Explosive welding test and performance study on composite sheet of tool-steel with Q235 carbon-steel[J]. Transactions of The China Welding Institution，2001，22(4): 47-50.

[10] 胡兰青, 卫英慧, 许并社, 等. 爆炸焊接钢/钢复合板 接合界面微观结构分析 [J].材料热处理学报, 2004, 25(1): 46-48. 
HU Lanqing, WEI Yinghui, XU Bingshe, et al. Analysis on the interface structure of clad steel plate produced by explosive welding[J]. Transactions of Metal Heat Treatment, 2004, 25(1): 46-48.

[11] ZHANG Nan, WANG Wenxian, CAO Xiaoqing, et al. The effect of annealing on the interface microstructure and mechanical characteristics of AZ31B/AA6061 composite plates fabricated by explosive welding[J]. Materials and Design, 2015, 65: 1100-1109.

[12] WU Jiaqi, WANG Wenxian, CAO Xiaoqing, et al. Interface bonding mechanism and mechanical behavior of AZ31B/TA2 composite plate cladded by explosive welding[J]. Rare Metal Materials and Engineering, 2017, 46(3): 640-645.

[13] 张婷婷, 王文先, 袁晓丹, 等. $\mathrm{Mg} / \mathrm{Al}$ 合金爆炸焊连接 及其界面接合机制[J]. 机械工程学报，2016，52(12): 52-58.

ZHANG Tingting, WANG Wenxian, YUAN Xiaodan, et al. Interface bonding mechanism of $\mathrm{Mg} / \mathrm{Al}$ alloy explosive welded[J]. Journal of Mechanical Engineering, 2016, 52(12): 52-58.

[14] 范敏郁, 郭训忠, 崔圣强, 等. 钛/铝/钛三层板的一次 爆炸复合 $[\mathrm{J}]$. 稀有金属材料与工程, 2017, 46(3):

770-776.

FAN Minyu, GUO Xunzhong, CUI Shengqiang, et al. One-step explosive bonding preparation of Titanium /Aluminum /Titanium laminates with three layers[J]. Rare Metal Materials and Engineering, 2017, 46(3): 770-776.

[15] AKBARI-MOUSAVI S A A, BARRETT L M , Al-HASSANI S T S, Explosive welding of metal plates[J]. Journal of Materials Processing Technology, 2008, 202(1-3): 224-239.

[16] WANG Xiao, ZHENG Yuanyuan, LIU Huixia, et al. Numerical study of the mechanism of explosive/impact welding using Smoothed Particle Hydrodynamics method[J]. Materials and Design, 2012, 35: 210-219.
[17] 袁晓丹. 铝/镁合金爆炸焊接层状复合界面形成机制及 数值模拟[D]. 太原: 太原理工大学, 2016 .

YUAN Xiaodan. The formation and simulation on the interface of $\mathrm{Al} / \mathrm{Mg}$ alloys laminated composite maded by explosive welding[D]. Taiyuan: Taiyuan University of Technology, 2016.

[18] 韩顺昌. 爆炸焊接界面相变与断口组织 $[\mathrm{M}]$. 北京：国 防工业出版社, 2011.

HAN Shunchang. Phase transformation and fractography of interface of explosive welding[M]. Beijing: Nation Defense Industry Press, 2011.

[19] 王耀华. 金属板材爆炸焊接研究与实践 [M]. 北京：国 防工业出版社, 2007.

WANG Yaohua. Research and practice of explosive welding of metal plates[M]. Beijing: Nation Defense Industry Press, 2007.

[20] XIA Hongbo , WANG Shaogang, BEN Haifeng. Microstructure and mechanical properties of $\mathrm{Ti} / \mathrm{Al}$ explosive cladding[J]. Materials and Design, 2014, 56: 1014-1019.

[21] BAHRANI A.S, BLACK T.J, CROSSLAND B. The mechanics of wave formation in explosive welding[J]. Proc. R. Soc. A 296, 1967: 123-136.

[22] AKBARI-MOUSAVI S A A, Al-HASSANI S T S. Finite element simulation of explosively-driven plate impact with application to explosive welding[J]. Materials and Design, 2008, 29: 1-19.

[23] SALEM S A L. Explosive welding of flat plates in free flight $[\mathrm{J}]$. International Journal of Impact Engineering, 1984, 2: 85-101.

作者简介: 魏屹, 男, 1992 年出生。主要研究方向为美基材料爆炸焊接。 E-mail: 651928434@qq.com

王永祯(通信作者), 男, 1968 年出生, 教授, 博士研究生导师。主要研 究方向为树脂材料改性及其光造型、石墨烯能源材料的结构表征技术。 E-mail: wyz1231@126.com 